\title{
Gross ways to live long: parasitic worms as an anti-inflammaging therapy?
}

Bruce Zhang and David Gems*

Institute of Healthy Ageing, and Research Department of Genetics, Evolution and Environment, University College London, London WC1E 6BT, UK.

\begin{abstract}
Evolutionary medicine argues that disease can arise because modern conditions do not match those in which we evolved. For example, a decline in exposure to commensal microbes and gastrointestinal helminths in developed countries has been linked to increased prevalence of allergic and autoimmune inflammatory disorders (the hygiene hypothesis). Accordingly, microbial and helminthic probiotic therapies have been explored as Darwinian treatments for these disorders. A further possibility is that loss of old friend commensals also increases the sterile, aging-associated inflammation known as inflammaging, that contributes to a range of age-related diseases, including cardiovascular disease, dementia and cancer. Interestingly, Crowe et al. (2020) recently reported that treatment with a secreted glycoprotein from a parasitic nematode can protect against murine aging by induction of anti-inflammatory mechanisms. Here, we explore the hypothesis that restorative helminth therapy would have anti-inflammaging effects. Could worm infections provide broad-spectrum protection against age-related disease?
\end{abstract}

Keywords: aging, anti-inflammaging helminth hypothesis, evolutionary medicine, helminth therapy, hygiene theory, inflammaging, old friends

\section{*Corresponding author:}

David Gems

Institute of Healthy Ageing, and Department of Genetics, Evolution and Environment

University College London

Gower Street

London WC1E 6BT, United Kingdom

david.gems@ucl.ac.uk 


\section{Introduction}

The aging process is the main cause of senescent multimorbidity, the co-occurrence of multiple chronic pathologies that includes the major diseases of late life. The geroscience approach views preventative intervention in the aging process as a means to simultaneously pre-empt the development of multiple age-related diseases (Austad, 2015). How does aging cause senescent multimorbidity? While there are clearly multiple contributory factors, one mechanism whose importance is becoming increasingly clear is inflammaging, the state of systemic, low-grade inflammation that increases with age, independently of attack by infectious pathogens (Franceschi et al., 2000). Such inflammation is a contributory factor in diverse age-related pathologies, including cardiovascular disease, dementia, cancer, chronic obstructive pulmonary disease (COPD), osteoporosis and age-related macular degeneration (Xia et al., 2016).

One cause of inflammaging is gut dysbiosis, an imbalance in the composition of the intestinal microbiome. Such imbalance can manifest as loss of immunomodulatory microbial species, and is exacerbated by pro-dysbiotic aspects of the modern lifestyle, including antibiotic usage and the so-called Western diet (Buford, 2017). One interpretation of the cause of such dysbiotic effects utilizes the "old friends" hypothesis (derived from the hygiene hypothesis (Strachan, 1989)). This argues that the human immune system evolved optimal function in a dirtier world and in the presence of various microbes and helminth parasites, whose removal leads to pathogenic immunological hyperactivity (Rook et al., 2004). Gut dysbiosis certainly does contribute to allergic and autoimmune inflammation prior to aging (Kamada et al., 2013). One corollary of the old friends hypothesis is that restoration of old friend species should attenuate such inflammatory hyperfunction and reduce disease. Indeed, fecal microbiota transfer from healthy donors has been suggested as a potential anti-inflammaging therapy (Franceschi and Campisi, 2014). While the possible role of the microbiome in inflammaging has engaged the imagination of biogerontologists, little consideration has been given to a possible similar role in aging of the macrobiome - in particular, helminth parasites, which include flukes, tapeworms and nematodes.

Parasitic helminths have infected humans throughout their evolutionary history. As a consequence, helminths have become master manipulators of our immune system in order to dodge host attack. Meanwhile we have evolved some levels of tolerance of their presence. The wisdom of sometimes putting up with helminths is illustrated by the pathologies that can result from overly aggressive anti-helminth responses, as in elephantiasis which can result from infection with the filarial nematode Wuchereria bancrofti (Figure 1a). Through such coevolution, normal human immune development and function is likely to have become dependent upon the presence of immunomodulatory helminths, as well as that of their microbial counterparts (Velasquez-Manoff, 2012). Indeed, like old friend 
microbes, reduced helminth infection in ultra-clean modern societies has been linked to increased rates of allergic and autoimmune inflammatory disease, while restorative helminth therapies can protect against these conditions (see next section). But does reduced helminth infection, like gut dysbiosis, also promote inflammaging in later life? And, consequently, could restorative helminth therapy reduce inflammaging and ameliorate the pathologies that it promotes (Figure 2a)?

Using a mouse model, Crowe et al. (2020) recently provided the first tantalising evidence that helminth therapy can protect against aging through anti-inflammatory mechanisms. In the light of their study, we have taken a conceptual research approach (surveying and repurposing published findings) (Blagosklonny and Pardee, 2002) to investigate the plausibility of anti-inflammaging helminth therapy. We will begin by describing the idea of helminth therapy in more detail.

a
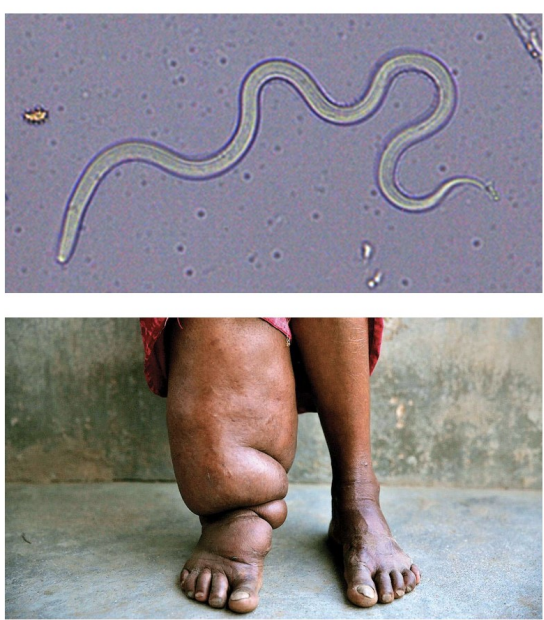

C

Acanthocheilonema viteae b

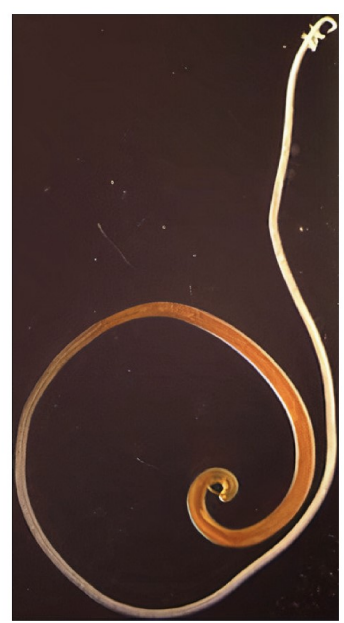

Asthma

Rheumatoid arthritis

Lupus

Gut permeability

Adiposity

Mortality

\section{Figure 1}

(a) The filarial nematode Wuchereria bancrofti (top) which can cause elephantiasis (bottom).

(b) The whipworm Trichuris suis. (c) The rodent filarial worm Acanthocheilonema viteae secretes a glycoprotein, ES-62, which is protective in murine models of asthma, rheumatoid arthritis and lupus. In addition, in a mouse model of high calorie diet-accelerated aging, ES62 administration prevented age-associated increases in gut permeability and adiposity, and increased longevity (Crowe et al., 2020). 


\section{Helminth therapy as a treatment for putative old friend loss disorders}

A variety of inflammatory disorders that afflict people prior to aging have been linked to a loss of old friend helminths. These include asthma, atopic eczema, inflammatory bowel disease, multiple sclerosis (MS), rheumatoid arthritis and type 1 diabetes (Maizels, 2020). For example, a study from Argentina found that accidental acquisition of gastrointestinal nematode infections not only caused a reduction in inflammatory cytokines, but alleviated disease symptoms in MS patients, and that clearance of parasites reversed these effects (Correale and Farez, 2007). In a similar vein, studies from Uganda found that hookworm infections in pregnant women confer protection against infantile atopic eczema, protection which is abrogated by anthelmintic treatment during pregnancy (Mpairwe et al., 2011, 2014).

More directly, a number of studies have documented beneficial effects of deliberate helminth infections, beginning with J.E. Turton's 1976 report in The Lancet which describes how maintaining an infection with the intestinal hookworm Necator americanus alleviated his allergies (Turton, 1976). More recently, clinical trials of helminth infection to treat patients with ulcerative colitis and Crohn's disease have yielded promising results (Varyani, Fleming and Maizels, 2017). Helminth therapy has also been tested extensively in rodents. For example, 20 out of 23 studies of the autoimmune encephalomyelitis mouse model of MS have reported helminth-induced reductions in disease severity (Charabati et al., 2020).

Unsurprisingly, the use of live helminths to treat old friend disorders remains controversial and giving oneself worms seems unwholesome, to say the least. More seriously, there are obvious safety worries: live helminth therapy might cause a resurgence of harmful infections in de-wormed countries or induce harmful side-effects. However, these concerns may be assuaged by using appropriate species (such as the pig whipworm Trichuris suis which does not reproduce in humans (Figure 1b)) and controlling dose size. Another strategy is to identify the mechanisms by which helminths manipulate host immunity and then apply those therapeutically. For example, administration of the glycoprotein ES-62 secreted by the rodent-infecting filarial nematode, Acanthocheilonema viteae, can recapitulate many of the immunomodulatory changes resulting from filarial infections and is protective in several pathological contexts (Figure 1c). Similarly, a variety of helminthderived antigens are protective in rodent models of multiple sclerosis (Dixit et al., 2017). Such approaches seem likely to yield safer and more precisely targeted therapeutics, and are certainly more palatable, though long-lasting, low level helminth infections might still represent cheaper and more efficient forms of therapy.

Taken together, helminth therapy currently shows promise as a means to treat inflammatory conditions such as allergy and autoimmunity (Figure 2a). But could it also protect against inflammaging? Could maintaining half a dozen hookworms in your bowel help slow aging? 


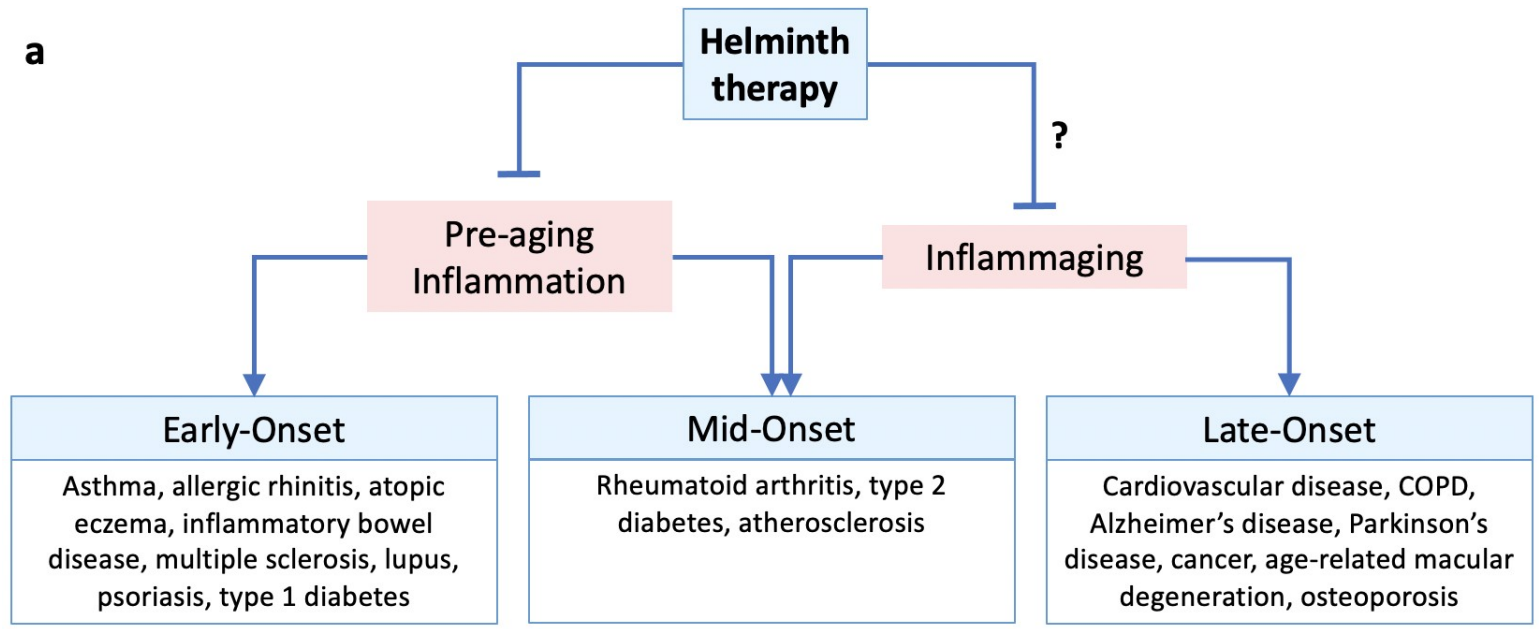

b

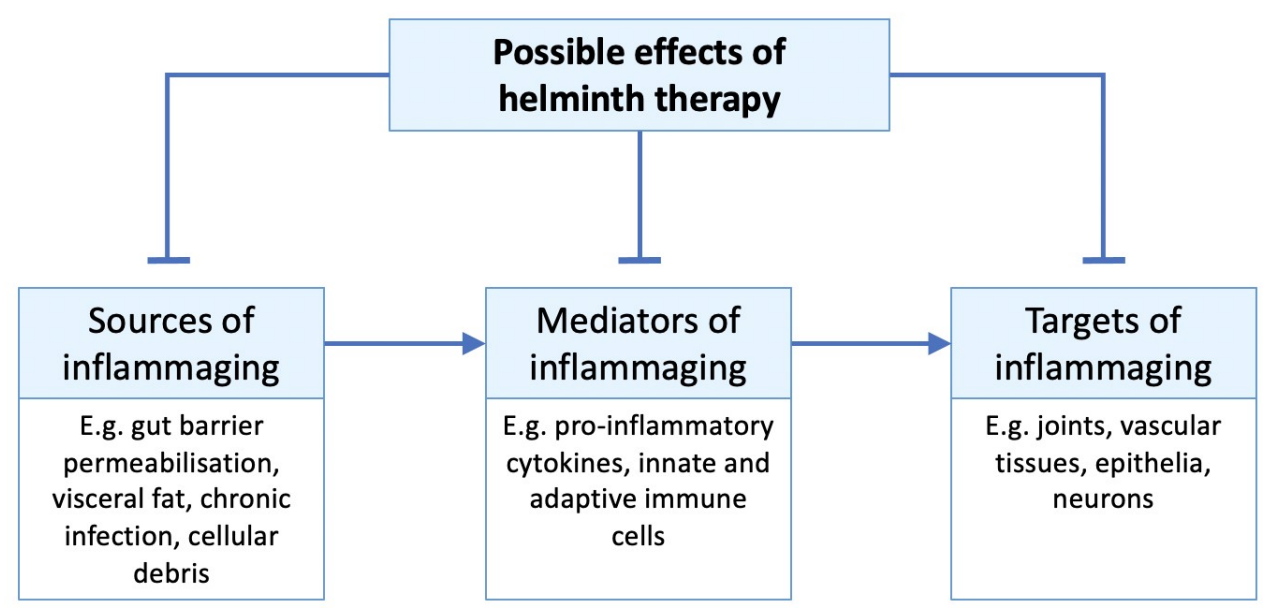

Figure 2

(a) Inflammatory disorders throughout the life course may be classed as early, mid and lateonset disorders. Pre-aging inflammation (e.g. allergic and autoimmune inflammation) contributes to early and some mid-onset disorders (all of which may persist into later life), while inflammaging appears later in life and contributes to late and some mid-onset disorders. Helminth therapy has been demonstrated to protect against early and mid-onset disorders by controlling pre-aging inflammation, but whether it can also protect against mid and late-onset disorders through anti-inflammaging mechanisms remains uncertain. We note that MS (age of onset 20-50 yr) and lupus (15-45 yr) are sometimes described as inflammaging-related (Sanai et al., 2016; Musella et al., 2018; Tsai et al., 2019). (b) Model of helminth-mediated attenuation of inflammaging acting at different pathophysiological stages. "Sources of inflammaging" are the mechanisms by which new inflammation is introduced; "mediators of inflammaging" are the components of existing inflammation itself; "targets of inflammaging" are the tissues damaged by the inflammation. Helminth therapy may target the inflammatory source and/or mediator and/or the damaged tissues. 


\section{Helminth therapy to counter inflammaging?}

Inflammaging can be detected as a sterile, persistent elevation of pro-inflammatory molecules in the blood, including cytokines such as IL-6 and TNF- $\alpha$ and acute phase proteins such as C-reactive protein (CRP). Interestingly, people infected by strongylid and filarial helminths have reduced levels of circulating pro-inflammatory cytokines and CRP (Aravindhan et al., 2010; George et al., 2014; Rajamanickam et al., 2019, 2020), and experimental helminth infection similarly reduces pro-inflammatory cytokines in human serum (Gaze et al., 2012). Furthermore, expulsion of helminths by anthelmintic medication induces elevated pro-inflammatory cytokine responsiveness in human blood (Bourke et al., 2013; Wammes et al., 2016). Thus, it is possible that helminth infections could attenuate the systemic inflammation that is inflammaging. But what about actual benefits in terms of improved health outcomes, such as reduced pathology and increased lifespan?

ES-62 is a $62 \mathrm{kDa}$ glycoprotein found in the excretory secretory products of the filarial nematode parasite $A$. viteae with anti-inflammatory properties. In the first study of its kind, Crowe et al. (2020) reported that weekly administration of ES-62 improved late-life health and increased lifespan (+12\%, mean lifespan) in a C57BL/6J mouse model of high calorie diet-accelerated aging. This suggests that the anti-inflammatory capacities of helminth therapy can exert protective effects even in later life.

Administration of ES-62 prevented the age-related decline in gut barrier integrity seen in high calorie diet-accelerated aging (Crowe et al., 2020). Of interest in the present context is that permeabilization of the gut lining can contribute to inflammaging, by allowing foreign antigens to leak into the blood and induce systemic immune activation (Thevaranjan et al., 2017; Ferrucci and Fabbri, 2018). But how could helminth therapy maintain gut barrier integrity? One way may be through the prevention of gut dysbiosis, a known contributor to inflammaging. Indeed, ES-62 prevented, and to some extent even reversed age-related decreases in Bacteroidetes: Firmicutes ratio and increases in Proteobacteria in the gut (Crowe et al., 2020), both of which are associated with inflammaging (Fransen et al., 2017). Another possible mode of action is helminth-induced production of IL-22, an essential cytokine for maintaining gut barrier integrity (Sonnenberg, Fouser and Artis, 2011). Thus, it is highly plausible that the extended lifespan was due to suppression of intestinal changes that contribute to inflammaging.

Administration of ES-62 also prevented adipocyte hypertrophy in gonadal visceral fat (Crowe et al., 2020). This, again, is notable given that adiposity is a major source of inflammaging. Adipose tissue, particularly visceral fat, is highly infiltrated by immune cells such as monocytes, B and T lymphocytes, mast cells and neutrophils, and is also a major site of senescent cell accumulation (Tchkonia et al., 2010; Mraz and Haluzik, 2014). Together, these adipocytes, immune cells and senescent cells secrete a potent and 
destructive cocktail of pro-inflammatory molecules, including TNF- $\alpha$, IL-6, IFN- $\gamma$ and leptin, which enter systemic circulation and contribute to the inflammaging phenotype. At the same time, anti-inflammatory mechanisms are reduced in adipose tissues, as shown by reduced numbers of eosinophils and of adiponectin secretion. In line with this, ES-62 administration reduced the decline in eosinophil number in visceral fat (Crowe et al., 2020).

Thus one potential mode of action of helminth therapy is suppression of adiposity. How does it achieve this? Nippostrongylus brasiliensis, a gastrointestinal roundworm of rodents, reduces adiposity apparently by reducing intestinal glucose absorption through altered STAT6 signaling (Moyat, Coakley and Harris, 2019). Whether humans show similar responses is unclear. One study of humans reported an inverse correlation between previous schistosome infection and central obesity, though adjustment for socio-economic status was not performed (Shen et al., 2015). Given the relationship between adiposity and inflammaging, helminth therapy might be of particular benefit to people who are overweight or obese.

The findings by Crowe et al. (2020) provide new support for the possibility that helminth therapy could protect against inflammaging. It would be interesting to know how ES-62 affects systemic markers of inflammation during inflammaging, which were not measured in the study, and also to examine effects on specific diseases linked to inflammaging. A number of earlier studies suggest that helminths can protect against such diseases, including rheumatoid arthritis (Ferrucci and Fabbri, 2018; Rea et al., 2018), type 2 diabetes (De Candia et al., 2019; Zuo et al., 2019), atherosclerosis (Zhuang and Lyga, 2014) and cancer (Leonardi et al., 2018; Zuo et al., 2019) (Figure 2a). We will consider the evidence for each of these in turn.

A prediction of the 'anti-inflammaging helminth' hypothesis is that in areas endemic for helminth infection, there should be lower rates of inflammaging-related disease, and there is some evidence for this. For example, in a region of Eastern India endemic for lymphatic filariasis infection, it was found that not one of out of 207 rheumatoid arthritis patients tested positive for circulating filarial nematode antigens; by contrast, $40 \%$ of 222 healthy controls were antigen positive (Panda et al., 2013). This is consistent with a protective role of helminth infection against rheumatoid arthritis. Indeed, experimental helminth infection or treatment with helminth antigens can protect against pathology in the collagen-induced arthritis (CIA) and MRL/lpr mouse models of rheumatoid arthritis (Smallwood et al., 2017; Langdon et al., 2019). Notably, ES-62-mediated protection against CIA was accompanied by reduced serum levels of the pro-inflammatory cytokine IL-17 (Pineda et al., 2012), consistent with protection against systemic inflammation.

Broadly similar findings have been reported for type 2 diabetes and atherosclerosis. Several epidemiological studies have reported an inverse relationship between the incidence 
of helminth infection and type 2 diabetes (Aravindhan et al., 2010; Chen et al., 2013; Hays et al., 2015), and also serum levels of the pro-inflammatory cytokines IL-6 and GM-CSF (Aravindhan et al., 2010); these studies controlled for age and BMI (and/or income). And again, experimental helminth infections protected mice from type 2 diabetes-associated states such as hyperglycemia and insulin resistance (Wu et al., 2011; Morimoto et al., 2016; Pace et al., 2018), while anthelmintic treatment elevated blood glucagon and insulin resistance, and several circulating pro-inflammatory cytokines (Tahapary et al., 2017; Rajamanickam et al., 2019, 2020). Regarding atherosclerosis, the burden of Opisthorchis felineus (cat liver fluke) in cadavers was found to be negatively correlated with the severity of aortic atherosclerosis (Magen et al., 2013). In atherosclerotic mouse models, infection with the blood fluke Schistosoma mansoni reduced atherosclerotic lesions by $50 \%$ (Doenhoff et al., 2002); similarly administration of Schistosoma egg antigens reduced atherosclerotic plaque size (Zhang et al., 2012; Wolfs et al., 2014).

There is also limited evidence that some cancers can be responsive to helminth therapy. The tapeworm Taenia crassiceps and its antigens have been found to attenuate and prevent colon tumorigenesis in mice through anti-inflammatory mechanisms (LeónCabrera et al., 2014; Callejas et al., 2019). However, in the same mouse model, the intestinal roundworm Heligmosomoides polygyrus was found to promote tumorigenesis (Pastille et al., 2017).

There is also some evidence linking helminth loss to other inflammaging-linked conditions. This includes COPD, given that helminths are known to suppress lung inflammation and to reduce levels of the pro-inflammatory cytokine IL-33, a driver of COPD pathology (Osbourn et al., 2017). IL-33 also promotes age-related macular degeneration (Xi et al., 2016), another late-life inflammaging-linked disease. Anecdotal evidence from homeusers of live helminth therapy suggest that old friend helminths may also provide protection against Parkinson's Disease and depression, although whether the latter includes geriatric depression is not known (Cheng et al., 2015). Both Parkinson's disease and geriatric depression are considered to be inflammaging-linked conditions (Teixeira et al., 2012; Calabrese et al., 2018).

\section{Perspectives}

There is clear evidence that an absence of helminth infection leads to increased incidence of inflammatory disorders, including some inflammaging-linked conditions, such as rheumatoid arthritis. Furthermore, experimental and clinical work has demonstrated the protective benefits of restorative helminth therapy against some inflammaging-linked diseases. Whether this reflects the ability of helminth therapy to reduce inflammaging remains uncertain, but clearly warrants further investigation. 
Given the role of inflammaging in numerous age-related diseases, further studies examining the effect of helminth therapy on multimorbidity would be especially useful. For example, it would be helpful to extend ongoing helminth therapy trials aimed at individual diseases to look at a broader range of conditions. The anti-inflammaging potential of helminth therapy could also be assessed by monitoring pro-inflammatory cytokine levels following administration of helminth therapy in patients with detectable inflammaging. Where live helminth therapy is used, infections can be easily terminated by anthelmintics should the need arise; conveniently, this would also provide additional data on the therapy's efficacy (i.e. does inflammaging then worsen?).

The possibility of anti-aging helminth therapy raises various questions. How do responses to such therapy change with age? Would higher or lower parasite (or antigen) levels be needed to take account of immunosenescence? To what extent do risks of helminth therapy increase in old age? What are optimal ages to apply such therapy to reduce inflammaging? Would helminth therapy act only in a preventative fashion (typical of anti-aging treatments), delaying the genesis or worsening of inflammaging-linked pathologies, or could it reverse existing disease symptoms? At least in murine rheumatoid arthritis and obesity, helminth therapy is both prophylactic and therapeutic (Yang et al., 2013; Rzepecka et al., 2015). Thus, it is possible that helminth therapy could, to some extent, reverse symptoms of aging as well as decelerate them.

Effective application of helminth therapy would also require a clear understanding of its specific anti-inflammaging mechanisms. Theoretically, helminths could counter inflammaging in several ways. For example, they could inhibit sources of inflammaging by preventing gut barrier permeabilization and obesity, neutralize existing inflammaging by increasing the proportion of anti-inflammatory to pro-inflammatory cytokines, or repair inflammaging-afflicted tissue damage e.g. through promotion of IL-22 secretion (Figure 2b).

\section{Conclusions}

It goes without saying that improvements in hygiene and elimination of helminth parasites have been of incalculable benefit to humanity. But a cost coupled to this benefit is abnormalities of immune function, specifically inflammatory hyperfunction. Available evidence suggests that restorative helminth therapies are effective against not only allergic and autoimmune inflammatory disorders, but also age-associated inflammation in later life, at least to some extent. Should this be confirmed, helminth therapy could provide protection against the wide spectrum of age-related diseases promoted by inflammaging. In the wake of successes during the last century in eliminating the evils of helminth infections, the time now seems propitious to explore further their possible benefits, particularly for our aging population - strange though this may sound. 


\section{Acknowledgements}

We would like to thank J. Crowe, J.M. Leeb and F.E. Lumb for useful discussion. Fig. 1a image (top, Wuchereria bancrofti) was adapted from Oxf. Med. Case Reports. 2015, Pandey, P., Dixit, A., Chandra, S., Tanwar, A., "Cytological diagnosis of bancroftian filariasis presented as a subcutaneous swelling in the cubital fossa: an unusual presentation" pp. 251-253 (2015). We thank Cheena Kapoor for permission to reproduce the Fig. 1a image of elephantiasis, and the Institute for Immunology, Free University Berlin for the Fig. 1b image of Trichuris suis. This work was supported by a Wellcome Trust Strategic Award (098565/Z/12/Z) and a Wellcome Trust Investigator Award (215574/Z/19/Z) to D. Gems.

\section{Conflict of interest}

The authors declare no competing financial interests.

\section{References}

Aravindhan, V., Mohan, V., Surendar, J., Muralidhara Rao, M., Pavankumar, N., Deepa, M., Rajagopalan, R., Kumaraswami, V., Nutman, T. B. and Babu, S. (2010) 'Decreased Prevalence of Lymphatic Filariasis among Diabetic Subjects Associated with a Diminished Pro-Inflammatory Cytokine Response (CURES 83)', PLoS Neglected Tropical Diseases. 4(6), e707.

Austad, S. N. (2016) 'The geroscience hypothesis: Is it possible to change the rate of aging?', In: Sierra F., Kohanski R. (eds) Advances in Geroscience. Springer, Cham.

Blagosklonny, M. V. and Pardee, A. B. (2002) 'Conceptual biology: unearthing the gems', Nature. 416(6879), 373.

Bourke, C. D., Nausch, N., Rujeni, N., Appleby, L. J., Mitchell, K. M., Midzi, N., Mduluza, T. and Mutapi, F. (2013) 'Integrated Analysis of Innate, Th1, Th2, Th17, and Regulatory Cytokines Identifies Changes in Immune Polarisation Following Treatment of Human Schistosomiasis', The Journal of Infectious Diseases, 208(1), 159-169.

Buford, T. W. (2017) '(Dis)Trust your gut: the gut microbiome in age-related inflammation, health, and disease', Microbiome. 5(1), 80.

Calabrese, V., Santoro, A., Monti, D., Crupi, R., Di Paola, R., Latteri, S., Cuzzocrea, S., Zappia, M., Giordano, J., Calabrese, E. J. and Franceschi, C. (2018) 'Aging and Parkinson's Disease: Inflammaging, neuroinflammation and biological remodeling as key factors in pathogenesis', Free Radical Biology and Medicine. 115, 80-91.

Callejas, B. E., Mendoza-Rodríguez, M. G., Villamar-Cruz, O., Reyes-Martínez, S., Sánchez-Barrera, C. A., Rodríguez-Sosa, M., Delgado-Buenrostro, N. L., Martínez-Saucedo, D., Chirino, Y. I., León-Cabrera, S. A., Pérez-Plasencia, C., Vaca-Paniagua, F., Arias-Romero, L. E. and Terrazas, L. I. (2019) 'Helminth-derived molecules inhibit colitis-associated colon cancer development through NF-kB and STAT3 regulation', International Journal of Cancer. 145(11), 3126-3139.

De Candia, P., Prattichizzo, F., Garavelli, S., De Rosa, V., Galgani, M., Rella, F. Di, Spagnuolo, M. I., Colamatteo, A., Fusco, C., Micillo, T., Bruzzaniti, S., Ceriello, A., Puca, A. A. and Matarese, G. (2019) 'Type 2 diabetes: How much of an autoimmune disease?', Frontiers in Endocrinology. 10, 451.

Charabati, M., Donkers, S. J., Kirkland, M. C. and Osborne, L. C. (2020) 'A critical analysis of helminth immunotherapy in multiple sclerosis', Multiple Sclerosis Journal. 26(12), 1448-1458.

Chen, Y., Lu, J., Huang, Y., Wang, T., Xu, Y., Xu, M., Li, M., Wang, W., Li, D., Bi, Y. and Ning, G. (2013) 'Association of Previous Schistosome Infection With Diabetes and 
Metabolic Syndrome: A Cross-Sectional Study in Rural China', The Journal of Clinical Endocrinology \& Metabolism. 98(2), E283-E287.

Cheng, A. M., Jaint, D., Thomas, S., Wilson, J. K. and Parker, W. (2015) 'Overcoming Evolutionary Mismatch by Self-Treatment with Helminths: Current Practices and Experience', Ashdin Publishing Journal of Evolutionary Medicine, 3, 22.

Correale, J. and Farez, M. (2007) 'Association between parasite infection and immune responses in multiple sclerosis', Annals of Neurology, 61(2), 97-108.

Crowe, J., Lumb, F. E., Doonan, J., Broussard, M., Tarafdar, A., Pineda, M. A., Landabaso, C., Mulvey, L., Hoskisson, P. A., Babayan, S. A., Selman, C., Harnett, W. and Harnett, M. M. (2020) 'The parasitic worm product ES-62 promotes health- And lifespan in a high calorie diet-accelerated mouse model of ageing', PLoS Pathogens. 16(3), e1008391.

Dixit, A., Tanaka, A., Greer, J. M. and Donnelly, S. (2017) 'Novel therapeutics for multiple sclerosis designed by parasitic worms', International Journal of Molecular Sciences. 18(10), 2141.

Doenhoff, M. J., Stanley, R. G., Griffiths, K. and Jackson, C. L. (2002) 'An anti-atherogenic effect of Schistosoma mansoni infections in mice associated with a parasite-induced lowering of blood total cholesterol', Parasitology. 125(5), 415-421.

Ferrucci, L. and Fabbri, E. (2018) 'Inflammageing: chronic inflammation in ageing, cardiovascular disease, and frailty', Nature Reviews Cardiology. 15(9), 505-522.

Franceschi, C., Bonafè, M., Valensin, S., Olivieri, F., De Luca, M., Ottaviani, E. and De Benedictis, G. (2000) 'Inflamm-aging: An Evolutionary Perspective on Immunosenescence', Annals of the New York Academy of Sciences. 908, 244-254.

Franceschi, C. and Campisi, J. (2014) 'Chronic inflammation (Inflammaging) and its potential contribution to age-associated diseases', Journals of Gerontology - Series A Biological Sciences and Medical Sciences. 69, S4-S9.

Fransen, F., van Beek, A. A., Borghuis, T., El Aidy, S., Hugenholtz, F., van der Gaast-de Jongh, C., Savelkoul, H. F. J., de Jonge, M. I., Boekschoten, M. V., Smidt, H., Faas, M. M. and de Vos, P. (2017) 'Aged gut microbiota contributes to systemical inflammaging after transfer to germ-free mice', Frontiers in Immunology. 8, 1385.

Gaze, S., McSorley, H. J., Daveson, J., Jones, D., Bethony, J. M., Oliveira, L. M., Speare, R., McCarthy, J. S., Engwerda, C. R., Croese, J. and Loukas, A. (2012) 'Characterising the mucosal and systemic immune responses to experimental human hookworm infection', PLoS Pathogens. 8(2), e1002520.

George, P. J., Kumar, N. P., Sridhar, R., Hanna, L. E., Nair, D., Banurekha, V. V., Nutman, T. B. and Babu, S. (2014) 'Coincident Helminth Infection Modulates Systemic Inflammation and Immune Activation in Active Pulmonary Tuberculosis', PLoS Neglected Tropical Diseases. 8(11), e3289.

Hays, R., Esterman, A., Giacomin, P., Loukas, A. and McDermott, R. (2015) 'Does Strongyloides stercoralis infection protect against type 2 diabetes in humans? Evidence from Australian Aboriginal adults', Diabetes Research and Clinical Practice. 107(3), 355-361.

Kamada, N., Seo, S. U., Chen, G. Y. and Núñez, G. (2013) 'Role of the gut microbiota in immunity and inflammatory disease', Nature Reviews Immunology. 13(5), 321-335.

Langdon, K., Phie, J., Thapa, C. B., Biros, E., Loukas, A. and Haleagrahara, N. (2019) 'Helminth-based therapies for rheumatoid arthritis: A systematic review and metaanalysis', International Immunopharmacology. 66, 366-372.

León-Cabrera, S., Callejas, B. E., Ledesma-Soto, Y., Coronel, J., Pérez-Plasencia, C., 
Gutiérrez-Cirlos, E. B., Ávila-Moreno, F., Rodríguez-Sosa, M., Hernández-Pando, R., Marquina-Castillo, B., Chirino, Y. I. and Terrazas, L. I. (2014) 'Extraintestinal helminth infection reduces the development of colitis-associated tumorigenesis', International Journal of Biological Sciences. 10(9), 948-956.

Leonardi, G. C., Accardi, G., Monastero, R., Nicoletti, F. and Libra, M. (2018) 'Ageing: From inflammation to cancer', Immunity and Ageing. 15, 1.

Magen, E., Bychkov, V., Ginovker, A. and Kashuba, E. (2013) 'Chronic Opisthorchis felineus infection attenuates atherosclerosis - An autopsy study', International Journal for Parasitology. 43(10), 819-824.

Maizels, R. M. (2020) 'Regulation of immunity and allergy by helminth parasites', Allergy. 75(3), 524-534.

Morimoto, M., Azuma, N., Kadowaki, H., Abe, T. and Suto, Y. (2016) 'Regulation of type 2 diabetes by helminth-induced Th2 immune response', Journal of Veterinary Medical Science. 78(12), 1855-1864.

Moyat, M., Coakley, G. and Harris, N. L. (2019) 'The interplay of type 2 immunity, helminth infection and the microbiota in regulating metabolism', Clinical \& Translational Immunology. 8(11), e01089.

Mpairwe, H., Ndibazza, J., Webb, E. L., Nampijja, M., Muhangi, L., Apule, B., Lule, S., Akurut, H., Kizito, D., Kakande, M., Jones, F. M., Fitzsimmons, C. M., Muwanga, M., Rodrigues, L. C., Dunne, D. W. and Elliott, A. M. (2014) 'Maternal hookworm modifies risk factors for childhood eczema: results from a birth cohort in Uganda', Pediatric Allergy and Immunology. 25(5), 481-488.

Mpairwe, H., Webb, E. L., Muhangi, L., Ndibazza, J., Akishule, D., Nampijja, M., Ngom-wegi, S., Tumusime, J., Jones, F. M., Fitzsimmons, C., Dunne, D. W., Muwanga, M., Rodrigues, L. C. and Elliott, A. M. (2011) 'Anthelminthic treatment during pregnancy is associated with increased risk of infantile eczema: Randomised-controlled trial results', Pediatric Allergy and Immunology, 22(3), 305-312.

Mraz, M. and Haluzik, M. (2014) 'The role of adipose tissue immune cells in obesity and lowgrade inflammation', Journal of Endocrinology. 222(3), 113-127.

Musella, A., Gentile, A., Rizzo, F. R., Vito, F. De, Fresegna, D., Bullitta, S., Vanni, V., Guadalupi, L., Bassi, M. S., Buttari, F., Centonze, D. and Mandolesi, G. (2018) 'Interplay between age and neuroinflammation in multiple sclerosis: Effects on motor and cognitive functions', Frontiers in Aging Neuroscience. 10, 238.

Osbourn, M., Soares, D. C., Vacca, F., Suzanne Cohen, E., Scott, I. C., Gregory, W. F., Smyth, D. J., Toivakka, M., Kemter, A. M., le Bihan, T., Wear, M., Hoving, D., Filbey, K. J., Hewitson, J. P., Henderson, H., Gonzàlez-Ciscar, A., Errington, C., Vermeren, S., Astier, A. L., Wallace, W. A., Schwarze, Ivens, A. C., Maizels, R. M., and McSorley, H. J. (2017) 'HpARI Protein Secreted by a Helminth Parasite Suppresses Interleukin-33', Immunity. 47(4), 739-751.e5.

Pace, F., Carvalho, B. M., Zanotto, T. M., Santos, A., Guadagnini, D., Silva, K. L. C., Mendes, M. C. S., Rocha, G. Z., Alegretti, S. M., Santos, G. A., Catharino, R. R., Paroni, R., Folli, F. and Saad, M. J. A. (2018) 'Helminth infection in mice improves insulin sensitivity via modulation of gut microbiota and fatty acid metabolism', Pharmacological Research. 132, 33-46.

Panda, A. K., Ravindran, B. and Das, B. K. (2013) 'Rheumatoid Arthritis Patients Are Free of Filarial Infection in an Area Where Filariasis is Endemic: Comment on the Article by Pineda et al', Arthritis \& Rheumatism. 65(5), 1402-1403.

Pastille, E., Frede, A., McSorley, H. J., Gräb, J., Adamczyk, A., Kollenda, S., Hansen, W., 
Epple, M., Buer, J., Maizels, R. M., Klopfleisch, R. and Westendorf, A. M. (2017) 'Intestinal helminth infection drives carcinogenesis in colitis-associated colon cancer', PLoS Pathogens. 13(9), e1006649.

Pineda, M. A., McGrath, M. A., Smith, P. C., Al-Riyami, L., Rzepecka, J., Gracie, J. A., Harnett, W. and Harnett, M. M. (2012) 'The parasitic helminth product ES-62 suppresses pathogenesis in collagen-induced arthritis by targeting the interleukin-17producing cellular network at multiple sites', Arthritis \& Rheumatism. 64(10), 31683178.

Rajamanickam, A., Munisankar, S., Bhootra, Y., Dolla, C., Thiruvengadam, K., Nutman, T. B. and Babu, S. (2019) 'Metabolic Consequences of Concomitant Strongyloides stercoralis Infection in Patients With Type 2 Diabetes Mellitus', Clinical Infectious Diseases, 69(4), 697-704.

Rajamanickam, A., Munisankar, S., Dolla, C., Menon, P. A., Thiruvengadam, K., Nutman, T. B. and Babu, S. (2020) 'Helminth infection modulates systemic pro-inflammatory cytokines and chemokines implicated in type 2 diabetes mellitus pathogenesis', PLOS Neglected Tropical Diseases. 14(3), e0008101.

Rea, I. M., Gibson, D. S., McGilligan, V., McNerlan, S. E., Denis Alexander, H. and Ross, O. A. (2018) 'Age and age-related diseases: Role of inflammation triggers and cytokines', Frontiers in Immunology. 9, 586.

Rook, G. A. W., Adams, V., Hunt, J., Palmer, R., Martinelli, R. and Brunet, L. R. (2004) 'Mycobacteria and other environmental organisms as immunomodulators for immunoregulatory disorders', Springer Seminars in Immunopathology. 25(3-4), 237255.

Rzepecka, J., Pineda, M. A., Al-Riyami, L., Rodgers, D. T., Huggan, J. K., Lumb, F. E., Khalaf, A. I., Meakin, P. J., Corbet, M., Ashford, M. L., Suckling, C. J., Harnett, M. M. and Harnett, W. (2015) 'Prophylactic and therapeutic treatment with a synthetic analogue of a parasitic worm product prevents experimental arthritis and inhibits IL$1 \beta$ production via NRF2-mediated counter-regulation of the inflammasome', Journal of Autoimmunity. 60, 59-73.

Sanai, S. A., Saini, V., Benedict, R. H., Zivadinov, R., Teter, B. E., Ramanathan, M. and Weinstock-Guttman, B. (2016) 'Aging and multiple sclerosis.', Multiple sclerosis. 22(6), 717-25.

Shen, S.-W., Lu, Y., Li, F., Shen, Z.-H., Xu, M., Yao, W.-F., Feng, Y.-B., Yun, J.-T., Wang, Y.-P., Ling, W., Qi, H.-J. and Tong, D.-X. (2015) 'The potential long-term effect of previous schistosome infection reduces the risk of metabolic syndrome among Chinese men', Parasite Immunology. 37(7), 333-339.

Smallwood, T. B., Giacomin, P. R., Loukas, A., Mulvenna, J. P., Clark, R. J. and Miles, J. J. (2017) 'Helminth immunomodulation in autoimmune disease', Frontiers in Immunology. 8, 453.

Sonnenberg, G. F., Fouser, L. A. and Artis, D. (2011) 'Border patrol: Regulation of immunity, inflammation and tissue homeostasis at barrier surfaces by IL-22', Nature Immunology. 12(5), 383-390.

Strachan, D. P. (1989) 'Hay fever, hygiene, and household size.', British Medical Journal. 299(6710), 1259-1260.

Tahapary, D. L., de Ruiter, K., Martin, I., A T Brienen, E., van Lieshout, L., M Cobbaert, C., Soewondo, P., Djuardi, Y., E Wiria, A., J Houwing-Duistermaat, J., Sartono, E., W A Smit, J., Yazdanbakhsh, M. and Supali, T. (2017) 'Effect of Anthelmintic Treatment on Insulin Resistance: A Cluster-Randomized, Placebo-Controlled Trial in Indonesia', 
Clinical Infectious Diseases, 65(5), 764-771.

Tchkonia, T., Morbeck, D. E., Von Zglinicki, T., Van Deursen, J., Lustgarten, J., Scrable, H., Khosla, S., Jensen, M. D. and Kirkland, J. L. (2010) 'Fat tissue, aging, and cellular senescence', Aging Cell. 9(5), 667-684.

Teixeira, A. L., Wieck, A., Diniz, B. S. and Bauer, M. E. (2012) 'Biomarkers in Mood Disorders Among the Elderly: Can They Contribute to Diagnosis and Prognosis?', Current Translational Geriatrics and Experimental Gerontology Reports. 1(2), 111120.

Thevaranjan, N., Puchta, A., Schulz, C., Naidoo, A., Szamosi, J. C., Verschoor, C. P., Loukov, D., Schenck, L. P., Jury, J., Foley, K. P., Schertzer, J. D., Larché, M. J., Davidson, D. J., Verdú, E. F., Surette, M. G. and Bowdish, D. M. E. (2017) 'AgeAssociated Microbial Dysbiosis Promotes Intestinal Permeability, Systemic Inflammation, and Macrophage Dysfunction', Cell Host and Microbe. 21(4), 455466.e4.

Tsai, C. Y., Shen, C. Y., Liao, H. T., Li, K. J., Lee, H. T., Lu, C. S., Wu, C. H., Kuo, Y. M., Hsieh, S. C. and Yu, C. L. (2019) 'Molecular and cellular bases of immunosenescence, inflammation, and cardiovascular complications mimicking "inflammaging" in patients with systemic lupus erythematosus', International Journal of Molecular Sciences. 20(16), 3878.

Turton, J. A. (1976) 'Letter: IgE, parasites, and allergy', The Lancet. 2(7987), 686.

Varyani, F., Fleming, J. O. and Maizels, R. M. (2017) 'Helminths in the gastrointestinal tract as modulators of immunity and pathology', American Journal of Physiology Gastrointestinal and Liver Physiology. 312(6), G537-G549.

Velasquez-Manoff, M. (2012) An Epidemic of Absence: A New Way of Understanding Allergies and Autoimmune Diseases. Scribner, New York.

Wammes, L. J., Hamid, F., Wiria, A. E., May, L., Kaisar, M. M. M., Prasetyani-Gieseler, M. A., Djuardi, Y., Wibowo, H., Kruize, Y. C. M., Verweij, J. J., De Jon, S. E., Tsonaka, R., Houwing-Duistermaat, J. J., Sartono, E., Luty, A. J. F., Supali, T. and Yazdanbakhsh, M. (2016) 'Community deworming alleviates geohelminth-induced immune hyporesponsiveness', Proceedings of the National Academy of Sciences of the United States of America., 113(44), 12526-12531.

Wolfs, I. M. J., Stöger, J. L., Goossens, P., Pöttgens, C., Gijbels, M. J. J., Wijnands, E., Van Der Vorst, E. P. C., Van Gorp, P., Beckers, L., Engel, D., Biessen, E. A. L., Kraal, G., Van Die, I., Donners, M. M. P. C. and De Winther, M. P. J. (2014) 'Reprogramming macrophages to an anti-inflammatory phenotype by helminth antigens reduces murine atherosclerosis', FASEB Journal. 28(1), 288-299.

Wu, D., Molofsky, A. B., Liang, H. E., Ricardo-Gonzalez, R. R., Jouihan, H. A., Bando, J. K., Chawla, A. and Locksley, R. M. (2011) 'Eosinophils sustain adipose alternatively activated macrophages associated with glucose homeostasis', Science. 332(6026), 243-247.

Xi, H., Katschke, K. J., Li, Y., Truong, T., Lee, W. P., Diehl, L., Rangell, L., Tao, J., Arceo, R., Eastham-Anderson, J., Hackney, J. A., Iglesias, A., Cote-Sierra, J., Elstrott, J., Weimer, R. M. and Van Lookeren Campagne, M. (2016) 'IL-33 amplifies an innate immune response in the degenerating retina', Journal of Experimental Medicine. 213(2), 189-207.

Xia, S., Zhang, X., Zheng, S., Khanabdali, R., Kalionis, B., Wu, J., Wan, W. and Tai, X. (2016) 'An Update on Inflamm-Aging: Mechanisms, Prevention, and Treatment', Journal of Immunology Research. 2016, 8426874. 
Yang, Z., Grinchuk, V., Smith, A., Qin, B., Bohl, J. A., Sun, R., Notari, L., Zhang, Z., Sesaki, H., Urban, J. F., Shea-Donohue, T. and Zhao, A. (2013) 'Parasitic nematode-induced modulation of body weight and associated metabolic dysfunction in mouse models of obesity', Infection and Immunity. 81(6), 1905-1914.

Zhang, H., Xing, Y., Kong, H., Dai, Y., He, W., Ge S., and Zhu, Y. (2012) '[Anti-atherogenic effect and its mechanisms of soluble egg antigen of Schistosomia japonicum in ApoE-/- mice]', Zhongguo Xue Xi Chong Bing Fang Zhi Za Zhi. 24(6), 654-658.

Zhuang, Y. and Lyga, J. (2014) 'Inflammaging in skin and other tissues - the roles of complement system and macrophage', Inflammation and Allergy - Drug Targets. 13(3), 153-161.

Zuo, L., Prather, E. R., Stetskiv, M., Garrison, D. E., Meade, J. R., Peace, T. I. and Zhou, T. (2019) 'Inflammaging and oxidative stress in human diseases: From molecular mechanisms to novel treatments', International Journal of Molecular Sciences. 20(18), 4472. 